\title{
Quality of Life (QOL) in Long-Term Survivors of Osteosarcoma*
}

\author{
Jae Do Kim, Jae Man Kwak, So Hak Chung ${ }^{\#}$ \\ Department of Orthopedic Surgery, Kosin University Gospel Hospital, Busan, Korea. \\ Email: \#shchung@kosin.ac.kr
}

Received December $2^{\text {nd }}, 2012$; revised January $4^{\text {th }}, 2013$; accepted January $11^{\text {th }}, 2013$

\begin{abstract}
We conducted this study to analyze the quality of life using the SF-36 health questionnaire and the MSTS scores depending on surgical method and the primary site of occurrence. On the surgical method, the limb salvage had more excellent outcomes (60.9) than the amputation (46.3). The tumor prosthetic replacement had notably higher scores (62.5) than any other methods. For the correlation coefficients on the MSTS and SF-36, the Physical Function had the highest degree of correlation. Excellent functions of the extremities would lead to a good quality of life.
\end{abstract}

Keywords: Osteosarcoma; Quality of Life; SF-36 Health Questionnaire

\section{Introduction}

With recent advances in treatment methods for osteosarcoma, a combination therapy of surgery, chemotherapy and radiotherapy has greatly improved long-term survival rate [1-3]. Traditional treatment method for patients with osteosarcoma mainly include limb-salvage surgery and amputation. Controversial opinions remain regarding the outcomes between the two methods. In recent years, however, an increased number of studies have reported that the treatment outcomes of limb-salvage surgery have improved [4-6]. Moreover, limb-salvage surgery has become increasingly common. Since the 2000s, its application has been almost popularized. The 5-year survival of patients underwent limb-salvage surgery has increased to $60 \%-70 \%$. On the other hand, orthopedic surgeons confront challenging problems related to limb-salvage surgery such as complications associated with the delayed chemotherapy (secondary malignancy or infertility) and postoperative complications such as infection and the mechanical failure or instability of the metal prosthesis [7-9]. Many studies have reported on the functional status of the extremities on the affected side in long-term survivors from osteosarcoma [10-13], however, there are insufficient about the quality of life [14-20]. So we conducted this study to evaluate the quality of life in longterm survivors from osteosarcoma. The goal of this study is to examine which limb salvage surgery is the most effective in improving the long-term survivors' quality of

\footnotetext{
*Note: This study was supported by a grant from Kosin University College of Medicine (2011).

${ }^{\#}$ Corresponding author.
}

life. Moreover, the correlation of limb function and QOL was studied to clarify the effects of limb function on the long term QOL.

\section{Patients and Methods}

\subsection{Patients}

Of the patients who were diagnosed with osteosarcoma at our medical institution from December 1989 to December 2007, 96 cases were long-term survivors who could be followed up during a minimum period of six years. Of those cases, 47 were available for a questionnaire survey. Informed consent was obtained from the patients before conducting the questionnaire. These cases had a mean age of 31.2 years, 25 were men and 22 were women. Of the 47 cases, 7 underwent amputation and the 40 had a limb-salvage surgery. Of the cases who underwent limb salvage surgery, 25 underwent tumor prosthesis replacement, 6 had allografts, and 5 had recycling autografts (2 extracorporeal irradiation and 3 pasteurization). According to anatomical region, there were 6 cases involving the upper extremities (3 cases of the proximal humerus, 2 cases of the distal radius and one case at the scapula) and 41 cases involving the lower extremities (23 cases of the distal femur, one case involving the femoral shaft, 13 cases involving the proximal tibia, two cases at the distal tibia, one case at the patella and one case at the calcaneus).

\subsection{Study Methods}

\subsubsection{Quality of Life Assessment}

As a tool for assessing the quality of life in long-term 
survivors, the SF-36 health questionnaire was performed using telephone and outpatient interviews. The questionnaire has been widely used worldwide, and its validity has been well demonstrated in studies. It is therefore considered a valid and reliable questionnaire [21]. It is a simple questionnaire consisting of only 36 questions. National standards of the Korea are also available [21]. For these reasons, the SF-36 was considered to be appropriate for the QOL assessment of long-term survivors of osteosarcoma and was selected for our evaluation. The SF-36 is composed of eight parameters: Physical functioning (PF), physical role (PR), bodily pain (BP), general health perceptions (GH), vitality (VT), social functioning (SF), emotional role (ER) and mental health $(\mathrm{MH})$. Following the questionnaire survey, scores on the eight parameters were converted into values that were adjusted to a mean of 50 points and a SD (SD: standard deviation) of 50 points. Based on a cut-off value of 50 points, the results of the current study could be interpreted and analyzed [21]. A total of 47 long-term survivors were divided into two groups: the amputation group $(n=7)$ and the limb-salvage surgery group $(n=40)$. This was followed by a comparison of the scores on the SF-36 health questionnaire survey between the two groups. In addition, we also classified the patients who underwent limb salvage surgery into cases of tumor prosthesis replacement, allo-bone graft, autologous bone graft (extracorporeal irradiation, pasteurization). Then, we evaluated a comparative analysis of these groups.

\subsubsection{Limb Function Assessment}

To assess the function of the extremities in long-term survivors, we used the American Musculoskeletal Tumor Society (MSTS) Scores [10]. The MSTS score is widely used for the evaluation of affected limb function. Lower limb function was evaluated according to six parameters: pain, function, emotional acceptance, supports, walking and gait, each of which was scored with a full mark of 5 (total of 30). Upper limb function was evaluated according to six parameters: pain, function, emotional acceptance, hand positioning, dexterity and lifting ability, each of which was scored with a full mark of 5 (total of 30). Thus, we compared the functions of extremities between the amputation group and the limb-salvage surgery group. In addition, by comparing the methods of limb-salvage surgery, we attempted to determine which is the best treatment option on limb function.

\subsubsection{The Correlation between the Quality of Life and the Functions of Extremities}

To identify the correlation between each parameters on the SF-36 health questionnaire survey and the MSTS Scores, we used the Pearson's correlation coefficient. Thus, we attempted to analyze the correlation between scores on the SF-36 health questionnaire and the MSTS.

\section{Result}

\subsection{The Quality of Life Assessment}

The eight parameters on the SF-36 health questionnaire survey, i.e., physical functioning (PF), physical role (RP), bodily pain (BP), general health perceptions(GH), vitality (VT), social functioning (SF), emotional role (RE) and mental health (MH), had a mean values of 60.5, 58.7, 61.3, 53.7, 56.2, 63.4, 60.7, 57.2, 57.2 and 63.9 points, respectively, all of which were higher than the national standard (Figure 1). Mean SF-36 scores between the limb salvage surgery group and the amputation group, were 61.0 and 47.0, respectively (Table 1). Following a comparison of the methods of limb salvage surgery,

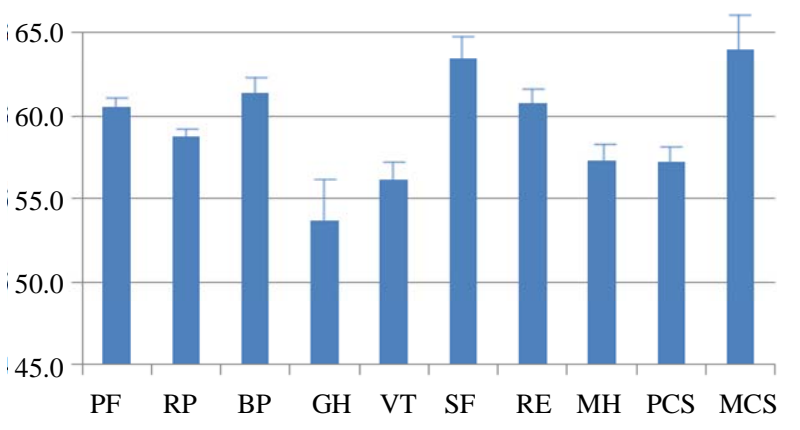

Figure 1. QOL of long-term survivors of osteosaroma. The QOL was assessed using 8 parameters. For each parameter, the mean of the national standard of the Japanese is adjusted to 50 points, and the standard deviation to 10 points. PF: physical functioning, RP: physical role, BP: bodily pain, GH: general health perceptions, VT: vitality, SF: social functioning, RE: emotional role, $\mathrm{MH}$ : mental health.

Table 1. Comparison of the QOL between the limb-sparing and amputation groups.

\begin{tabular}{ccccc}
\hline Parameter & \multicolumn{2}{c}{ Limb-sparing } & \multicolumn{2}{c}{ Amputation } \\
\hline & \multicolumn{2}{c}{$(40$ pts. $)$} & \multicolumn{2}{c}{ pts. } \\
PF & Mean & SD & Mean & SD \\
RP & 62.8 & 0.52 & 42.5 & 1.45 \\
BP & 60.2 & 0.28 & 47.0 & 0.03 \\
GH & 63.2 & 0.66 & 51.6 & 1.41 \\
VT & 55.6 & 1.73 & 40.2 & 2.19 \\
SF & 57.8 & 1.03 & 46.7 & 0.12 \\
RE & 65.0 & 1.23 & 51.4 & 1.35 \\
MH & 63.1 & 0.62 & 46.8 & 0.09 \\
PCS & 59.2 & 0.59 & 44.2 & 0.91 \\
MCS & 58.8 & 0.73 & 47.0 & 0.03 \\
\hline
\end{tabular}

SD: standard deviation, PF: physical functioning, RP: physical role, BP: bodily pain, GH: general health perceptions, VT: vitality, SF: social functioning, RE: emotional role, MH: mental health. 
mean SF-36 scores were 63.1 in the tumor prosthetic replacement group, 58.4 in the allo bone graft group, 51.6 in the pasteurization group, and 60.7 in the extracorporeal irradiation group.

These results indicate that the tumor prosthetic replacement group of limb salvage surgery was the highest score(Table 2). According to primary sites, mean SF-36 scores were 69.3 in the patella, 67.4 in the humerus, 66.9 in the distal femur and 66.7 in the proximal tibia.

\subsection{The Assessment of the Functions of the Extremities}

Overall, mean MSTS scores, a measure of the function of the extremities, were 23.8 (15 - 28) in a total of 47 cases. In addition, the average score in the limb salvage surgery group was 24.35 (15 - 28) and 21.28 (19 - 23) in the amputation group. The mean was 24.5 in the tumor prosthetic replacement group, 25 in the allo bone graft group, 23 in the pasteurization group, and 21.5 in the extracorporeal irradiation group of recycling autologous bone graft.

\subsection{The Correlation between the Quality of Life and the MSTS Scores}

To identify the correlation between the quality of life and the MSTS scores, we used the Pearson's correlation coefficient factor. Following an analysis of the correlation with eight parameters on the SF-36 health questionnaire, the physical component summary (PCS) had a Pearson's correlation coefficient of 0.62 . Of these, physical functioning (PF) had the highest Pearson's correlation coefficient (0.86). In addition, the mental component summary

Table 2. SF-36 according to type of surgery.

\begin{tabular}{ccccc}
\hline & Tumor prosthesis & Pausteurization & IORBG & Allograft \\
\hline PF & $(25$ pts. & $(3$ pts. & $(2$ pts. $)$ & (6 pts.) \\
RP & 64.60 & 46.67 & 57.50 & 65.83 \\
BP & 61.90 & 50.00 & 56.25 & 57.91 \\
GH & 56.80 & 55.83 & 65.00 & 63.33 \\
VT & 59.76 & 45.00 & 52.50 & 55.83 \\
SF & 66.51 & 47.93 & 59.40 & 54.18 \\
RE & 66.26 & 54.17 & 75.00 & 59.53 \\
MH & 61.58 & 58.33 & 62.50 & 54.12 \\
PCS & 61.16 & 55.00 & 57.50 & 56.67 \\
MCS & 69.30 & 49.37 & 57.80 & 54.73 \\
\hline
\end{tabular}

PF: physical functioning, RP: physical role, BP: bodily pain, GH: general health perceptions, VT: vitality, SF: social functioning, RE: emotional role, MH: mental health, PCS: physical component summary, MCS: mental component summay.
(MCS) had a Pearson's correlation coefficient of 0.51 , corresponding to a relatively lower degree of correlation (Table 3).

\section{Discussion}

Limb salvage surgery is superior to amputation from both psychological and functional scores. With the advancement of combination chemotherapy and radiotherapy, treatment outcomes have improved rates of long term survival. With the improved survival rate, the number of long-term survivors has also increased. This has led to an increased interest in the quality of life. To date, various methods have been examined and applied in efforts to measure patients' quality of life [14-18]. However, there are no established standards for measuring quality of life $[13,19]$. Total care is necessary for osteosarcoma patients, many of whom are children, and a standard QOL assessment system must also be established for the assessment of total care. For the future, we aim to establish a standard QOL assessment system and to perform multi-institutional collaborative studies involving a greater number of patients. Currently available tools for measuring quality of life are disease-specific or generic health profile tools. Of these, the disease-specific tools are disadvantageous in that interpretation of their results can vary depending on which methods are used, although these tools have a high sensitivity to quality of life in specific diseases because they were designed to respond to disease-specific questions. The reliability and validity of many of the generic health profile tools have been demonstrated in many studies. Overall, they are useful to measure the quality of life. They are also advantageous in that they are useful to evaluate quality of life in comparison with other disease groups. Of these

Table 3. Correlation between the MSTS score and SF-36.

\begin{tabular}{cc}
\hline Parameter & CC \\
\hline PF & 0.86 \\
BP & 0.60 \\
GH & 0.56 \\
VT & 0.62 \\
SF & 0.57 \\
RE & 0.56 \\
MH & 0.73 \\
PCS & 0.60 \\
MCS & 0.62 \\
\hline
\end{tabular}

CC: correlation coefficient, PF: physical functioning, RP: physical role, BP: bodily pain, GH: general health perceptions, VT: vitality, SF: social functioning, RE: emotional role, MH: mental health, PCS: physical component summary, MCS: mental component summary, statistically significant. 
generic tools, we used the SF-36 health questionnaire for the current study. It has been translated in many different languages and customized to each country. In addition, its translated versions have been validated and revised. Based on the telephone interviews and self-reporting questionnaires, it can be used in a large patient population and can give an unbiased comparison of the psychological and physical factors, as well as evaluate quality of life in comparison with other disease groups.

We evaluated the quality of life in long-term survivors from osteosarcoma in patients at our medical institution. The mean quality of life scores were higher on a total of eight parameters as compared with the standard values on the SF-36 health questionnaire. But 50.5\% of eligible patients enrolled in the current study were not cooperative with the questionnaire survey, which might have affected our results. This should be considered. A majority of eligible patients who were cooperative with the questionnaire survey had positive responses. These positive attitudes might have contributed to an improved measure of quality of life.

All eight parameters had higher scores in the limb salvage surgery group than in the amputation group. To date, studies have also been conducted to compare the degree of quality of life between patients undergoing limb salvage surgery or amputation. Many of these studies have shown that there is no significant difference in the longterm quality of life between the two groups $[15-18,20]$. In contrast to these reports, our results showed that the degree of long term quality of life was higher in the limb salvage surgery group compared with the amputation group.

Some studies have reported that the MSTS score did not correlate with QOL $[19,22]$. But in this study, there was a significant correlation between the MSTS scores and the physical component summary (PCS) on the SF-36 health questionnaire. But there was lower correlation between the MSTS scores and the mental component summary (MCS) on the SF-36 health questionnaire. These results indicate that the mental health quality of life is not wholly dependent on the function of the extremities. It was shown, however, that the function of the extremities has a significant correlation with the quality of life to an extent. Previous studies have shown that limb-salvage surgery produces a variety of complications that can lead to poor quality of life. Our results are of significance, however, in that they showed the limb-salvage surgery restored the function of the extremities and this led to an improved quality of life.

\section{Conclusion}

We had the satisfactory results on all the eight parameter of the SF-36 health questionnaire. Despite many compli- cations of the limb salvage surgery, the tumor prosthetic replacement is considered an effective surgical method in improving the quality of life. Excellent functions of the extremities would lead to a good quality of life.

\section{REFERENCES}

[1] J. S. Whelan, et al., “Osteosarcoma,” European Journal of Cancer, Vol. 33, No. 10, 1997, pp. 1611-1619. doi:10.1016/S0959-8049(97)00251-7

[2] T. Yonemoto, S. Tatezaki, T. Ishii, T. Satoh, H. Kimura and N. Iwai, "Prognosis of Osteosarcoma with Pulmonary Metastases at Initial Presentation Is Not Dismal,” Clinical Orthopaedics and Related Research, Vol. 349, 1998, pp. 194-199. doi:10.1097/00003086-199804000-00024

[3] T. Yonemoto, S. Tatezaki, T. Ishii, K. Osato and T. Takenouchi, "Long-Term Survival after Surgical Removal of Solitary Brain Metastasis from Osteosarcoma," International Journal of Clinical Oncology, Vol. 8, No. 5, 2003, pp. 340-342. doi:10.1007/s10147-003-0341-9

[4] W. Enneking, et al., "Thirty Years of Limb Salvage," La Chirurgia degliorgani di movimento, Vol. 88, No. 4, 2003, pp. 321-325.

[5] T. Yonemoto, S. Tatezaki and T. Ishii, "Knee RotationPlasty for Malignant Musculoskeletal Tumors Occurring around the Knee Joint," Anticancer Research, Vol. 21, No. 1B, 2001, pp. 717-722.

[6] H. Tsuchiya, M. E. Abdel-Wanis and K. Tomita, "Biological Reconstruction after Excision of Juxta-Articular Osteosarcoma around the Knee: A New Classification System," Anticancer Research, Vol. 26, No. 1B, 2006, pp. 447-453.

[7] T. Yonemoto, S. Tatezaki, T. Ishii, T. Satoh and M. Inoue, "Two Cases of Osteosarcoma Occurring as Second Malignancy of Childhood Cancer," Anticancer Research, Vol. 19, No. 6C, 1999, pp. 5563-5566.

[8] T. Yonemoto, S. Tatezaki, T. Ishii and Y. Hagiwara, "Marriage and Fertility in Long-Term Survivors of High Grade Osteosarcoma,” American Journal of Clinical Oncology, Vol. 26, No. 5, 2003, pp. 513-516. doi:10.1097/01.coc.0000037666.38888.A5

[9] T. Yonemoto, S. Tatezaki, T. Ishii, Y. Hagiwara and M. Inoue, "Multiple Primary Cancers in Patients with Osteosarcoma: The Influence of Anticancer Drugs and Genetic Factors," American Journal of Clinical Oncology, Vol. 27, No. 3, 2004, pp. 220-224. doi:10.1097/01.COC.0000054534.43117.76

[10] W. F. Enneking, W. Dunham, M. C. Gebhardt, M. Malawar and D. J. Pritchard, "A System for the Functional Evaluation of Reconstructive Procedures after Surgical Treatment of Tumors of the Musculoskeletal System," Clinical Orthopaedics and Related Research, Vol. 286, 1993, pp. 241-246.

[11] A. J. Renard, R. P. Veth, H. W. Schreuder, C. J. van Loon, H. S. Koops and J. R. van Horn, "Function and Complications after Ablative and Limb-Salvage Therapy in Lower Extremity Sarcoma Of Bone,” Journal of Surgical Oncology, Vol. 73, No. 4, 2000, pp. 198-205. 
doi:10.1002/(SICI)1096-9098(200004)73:4<198::AID-JS O3>3.0.CO;2-X

[12] R. Nagarajan, J. P. Neglia, D. R. Clohisy and L. L. Robison, "Limb Salvage and Amputation in Survivors of Pediatric Lower-Extremity Bone Tumors: What Are the Long-Term Implications?” Journal of Clinical Oncology, Vol. 20, No. 22, 2002, pp. 4493-4501. doi:10.1200/JCO.2002.09.006

[13] P. J. Pakulis, N. L. Young and A. M. Davis, "Evaluating Physical Function in an Adolescent Bone Tumor Population,” Pediatric Blood \& Cancer, Vol. 45, No. 5, 2005, pp. 635-643. doi:10.1002/pbc.20383

[14] R. Nagarajan, D. R. Clohisy, J. P. Neglia, Y. Yasui, P. A. Mitby, C. Sklar, J. Z. Finklestein, M. Greenberg, G. H. Reaman, L. Zeltzer and L. L. Robison, "Function and Quality-Of-Life of Survivors of Pelvic and Lower Extremity Osteosarcoma and Ewing's Sarcoma: The Childhood Cancer Survivor Study,” British Journal of Cancer, Vol. 91, 2004, pp. 1858-1865. doi:10.1038/sj.bjc.6602220

[15] A. Postma, A. Kingma, J. H. De Ruiter, H. SchraffordtKoops, R. P. Veth, L. N. Goeken and W. A. Kamps, "Quality of Life in Bone Tumor Patients Comparing Limb Salvage and Amputation of the Lower Extremity," Journal of Surgical Oncology, Vol. 51, No. 1, 1992, pp. 47-51. doi:10.1002/jso.2930510113

[16] B. T. Rougraff, M. A. Simon, J. S. Kneisl, D. B. Greenberg and H. J. Mankin, "Limb Salvage Compared with Amputation for Osteosarcoma of the Distal End of the Femur. A Long-Term Oncological, Functional, and Quality-Of-Life Study,” The Journal of Bone \& Joint Surgery, Vol. 76A, No. 5, 1994, pp. 649-656.
[17] Y. Refaat, J. Gunnoe, F. J. Hornicek and H. J. Mankin, "Comparison of Quality of Life after Amputation or Limb Salvage,” Clinical Orthopaedics and Related Research, Vol. 397, 2002, pp. 298-305. doi:10.1097/00003086-200204000-00034

[18] A. Zahlten-Hinguranage, L. Bernd, V. Ewerbeck and D. Sabo, "Equal Quality of Life after Limb-Sparing or Ablative Surgery for Lower Extremity Sarcomas," British Journal of Cancer, Vol. 91, No. 6, 2004, pp. 1012-1014.

[19] V. G. Marchese, S. Ogle, R. B. Womer, J. Dormans and J. P. Ginsberg, "An Examination of Outcome Measures to Assess Functional Mobility in Childhood Survivors of Osteosarcoma,” Pediatric Blood \& Cancer, Vol. 42, No. 1, 2004, pp. 41-45. doi:10.1002/pbc.10462

[20] T. Akahane, T. Shimizu, K. Isobe, Y. Yoshimura, F. Fujioka and H. Kato, "Evaluation of Postoperative General Quality of Life for Patients with Osteosarcoma around the Knee Joint,” Journal of Pediatric Orthopaedics, Vol. 16, No. 4, 2007, pp. 269-272. doi:10.1097/BPB.0b013e3280925670

[21] C.-W. Han and E.-J. Lee, "Development of the Korean Version of Short-Form 36-Item Health Survey: Health Related QOL of Healthy Elderly People and Elderly Patients in Korea," The Tohoku Journal of Experimental Medicine, Vol. 203, No. 3, 2004, pp. 189-194. doi:10.1620/tjem.203.189

[22] T. Yonemoto, T. Ishii, Y. Takeuchi, K. Kimura, Y. Hagiwara, S. Iwata and S.-I. Tatezaki, "Evaluation of Quality of Life (QOL) in Long-term Survivors of High-grade Osteosarcoma: A Japanese Single Center Experience,” Anticancer Research, Vol. 27, 2007, pp. 3621-3624. 